\section{(2) OPEN ACCESS}

\title{
Multidimensional analyses reveal distinct immune microenvironment in hepatitis B virus-related hepatocellular carcinoma
}

\author{
Chun Jye Lim, ${ }^{1}$ Yun Hua Lee, ${ }^{1}$ Lu Pan, ${ }^{1}$ Liyun Lai, ${ }^{1}$ Camillus Chua, ${ }^{1}$ Martin Wasser, ${ }^{1}$ \\ Tony Kiat Hon Lim, ${ }^{2,3}$ Joe Yeong, ${ }^{2,4}$ Han Chong Toh, ${ }^{3,5}$ Ser Yee Lee, ${ }^{3,5,6}$ \\ Chung Yip Chan, ${ }^{3,5,6}$ Brian KP Goh, ${ }^{3,5,6}$ Alexander Chung, ${ }^{3,5,6}$ Mathias Heikenwälder, ${ }^{7}$ \\ Irene OL Ng, ${ }^{8}$ Pierce Chow, ${ }^{3,5,6}$ Salvatore Albani, ${ }^{1}$ Valerie Chew ${ }^{1}$
}

- Additional material is published online only. To view, please visit the journal online (http://dx.doi.org/10.1136/ gutjnl-2018-316510).

For numbered affiliations see end of article.

\section{Correspondence to}

Dr Valerie Chew, Translational Immunology Institute,

SingHealth- DukeNUS Academic Medical Centre, Singapore 169856, Singapore; valerie.chew.s.p@singhealth. com.sg

$\mathrm{CJL}$ and YHL contributed equally.

SA and VC contributed equally.

Received 26 March 2018 Revised 30 May 2018 Accepted 1 June 2018 Published Online First 3 July 2018
Check for updates

To cite: Lim CJ, Lee YH, Pan L, et al. Gut

2019;68:916-927.

\section{ABSTRACT}

Background and aims Chronic inflammation induced by chronic hepatitis B virus (HBV) infection increases the risk of hepatocellular carcinoma (HCC). However, little is known about the immune landscape of HBV-related $\mathrm{HCC}$ and its influence on the design of effective cancer immunotherapeutics.

Methods We interrogated the immune microenvironments of HBV-related HCC and non-viralrelated HCC using immunohistochemistry and cytometry by time-of-flight (CyTOF). On identifying unique immune subsets enriched in HBV-related HCC, we further interrogated their phenotypes and functions using next-generation sequencing (NGS) and in vitro T-cell proliferation assays.

Results In-depth interrogation of the immune landscapes showed that regulatory $T$ cells $\left(T_{\text {REG }}\right)$ and $\mathrm{CD}^{+}$resident memory $T$ cells $\left(T_{\mathrm{RM}}\right)$ were enriched in HBV-related HCC, whereas Tim- $3^{+} \mathrm{CD}^{+} \mathrm{T}$ cells and CD244 ${ }^{+}$natural killer cells were enriched in non-viralrelated HCC. NGS of isolated $T_{R E G}$ and $T_{\text {RM }}$ from HBVrelated HCC and non-viral-related HCC identified distinct functional signatures associated with T-cell receptor signalling, T-cell costimulation, antigen presentation and programmed cell death protein 1 (PD-1) signalling. $T_{\text {REG }}$ and $T_{R M}$ from HBV-related HCC expressed more PD-1 and were functionally more suppressive and exhausted than those from non-virus-related HCC. Furthermore, immunosuppression by PD- $1^{+} \mathrm{T}_{\text {REG }}$ could be reversed with anti-PD-1 blockade. Using multiplexed tissue immunofluorescence, we further demonstrated that $T_{\text {REG }}$ and $T_{R M}$ contributed to overall patient survival: $T_{\text {REG }}$ were associated with a poor prognosis and $T_{R M}$ were associated with a good prognosis in HCC.

Conclusion We have shown that the HBV-related $\mathrm{HCC}$ microenvironment is more immunosuppressive and exhausted than the non-viral-related HCC microenvironment. Such in-depth understanding has important implications in disease management and appropriate application of immunotherapeutics.

\section{INTRODUCTION}

Hepatocellular carcinoma (HCC) is one of the most common cancers among males and the third most common cause of cancer-associated death, globally.

\section{Significance of this study}

What is already known on this subject?

- The tumour microenvironment (TME) of HBVrelated HCC has mostly been studied in the context of one particular immune subset or by comparing the HBV-related HCC with livers from patients with chronic HBV infection or healthy individuals.

- A comprehensive and multidimensional analysis focusing on HBV-related HCC versus non-viralrelated $\mathrm{HCC}$ is lacking.

What are the new findings?

- We analysed the TME of HBV-related HCC in comparison to non-viral-related HCC using CyTOF and NGS.

- We identified distinct immune subsets enriched in both HCC aetiologies, and found that the HBV-related HCC immune landscape consisted of more immunosuppressive and exhausted phenotypes.

- $\mathrm{T}_{\mathrm{REG}}$ and $\mathrm{rT}_{\mathrm{RM}}$ that are enriched in HBV-related $\mathrm{HCC}$ are associated with overall patient survival in opposing directions.

How might it impact on clinical practice in the foreseeable future?

- An in-depth understanding of the TME in HBV-related versus non-viral-related HCC will allow us to design rational combinatory immunotherapy specific for each aetiology.

- Novel immunotherapeutics that target unique pathways in either HBV-related HCC or nonviral-related HCC could be designed for better disease management.

HBV infection is the single most common cause of HCC; up to 400 million individuals worldwide are living with a chronic HBV infection and are at high risk of developing HCC, with the majority of cases found in Asia and Africa. ${ }^{12}$

The different aetiologies of HCC, particularly those arising from chronic viral hepatitis, are well described, but their impact on the tumour microenvironment (TME) and the subsequent implications 
on disease management are not well understood. Much research in this area has focused on one single immune subset or has compared the immune profile of viral-related HCC with healthy livers or livers exposed to chronic hepatitis virus infection. ${ }^{34} \mathrm{~A}$ comprehensive study on the distinct immune landscapes and the underlying mechanisms in HCC with or without chronic HBV infection remains unexplored. There is a general consensus that the immune system is pivotal in determining the clinical fate of patients with HCC. 5 Viruses may also reprogramme their immune microenvironment to induce immunosuppression, peripheral tolerance during chronic infection and ultimately, tumourigenesis. ${ }^{6}$ Hence, we consider a better understanding of the immune landscapes in HBV-related and non-viral-related HCC will greatly help us evaluate the impact of TME in disease progression and prognosis.

Understanding the composition of the immune landscape within a cancer is also central to our understanding of responsiveness to immunotherapy. Recent breakthroughs in cancer immunotherapy, especially the development of immune checkpoint inhibitors, have yielded promising outcomes in clinical trials for multiple cancers, including HCC. ${ }^{78}$ Despite these advances, fundamental differences in patient responsiveness to such therapies are unexplained. Some have attributed these differences to the mutational burden of the tumour ${ }^{9}$ or the presence of neo-antigen-specific T cells, ${ }^{10}$ whereas others have highlighted the importance of tumour-immune infiltrates. ${ }^{11}$ Large-scale transcriptomic and immunogenetics analyses stratifying cancer immune landscapes in the attempt to shed light on this area were also recently reported. ${ }^{12} 13$ The response rate to the checkpoint inhibitor, nivolumab (anti-PD-1 monoclonal antibody) for HCC is 15\%-20\%, and no marked difference between HBV-related or non-viral-related HCC has been identified. ${ }^{14}$ Little is known regarding the mechanisms underlying the patient response to treatment. Over recent years, we and others have used CyTOF that simultaneously analyse single cells for $\leq 41$ cellular parameters to immunophenotype a number of diseases, including HCC. ${ }^{15-17}$ Such multidimensional analyses have helped us gain a deep understanding of the TME and its influence on disease progression and response to immunotherapy.

Here, we combined CyTOF, NGS and multiplexed tissue immunoflorescence (IF) to compare the immune landscapes between HBV-related HCC and non-viral-related HCC. By this method, we identified several distinct immune populations specific to $\mathrm{HBV}$-related $\mathrm{HCC}$ with unique functionality, and determined their impact on the overall survival (OS) of affected patients.

\section{MATERIALS AND METHODS Patients}

Fresh tumour tissues, adjacent non-tumour tissues and blood samples were collected from 23 patients with HCC who underwent surgical resection at the National Cancer Center Singapore or Singapore General Hospital (SGH) (online supplementary table S1). Tumour-infiltrating leukocytes (TILs) and non-tumour-infiltrating leukocytes (NILs) were isolated using $100 \mathrm{U} /$ $\mathrm{mL}$ collagen intravenously. Peripheral blood mononuclear cells (PBMCs) were isolated from preoperative blood samples using Ficoll-Pague Plus (GE Healthcare), as previously described. ${ }^{18}$ Formalin-fixed and paraffin-embedded (FFPE) tissue-microarray (TMA) slides were obtained from an independent HCC cohort $(n=102)$ from the Department of Pathology, SGH (online supplementary table S2). A further set of FFPE tissues of resected HCC $(n=116$ patients) were collected from SGH $(n=37)$; the Queen Mary Hospital, Hong Kong $(n=42)$ and the University Hospital
Zurich, Switzerland $(\mathrm{n}=37)$ (online supplementary table S3). Patient consents were obtained following the guidelines of each Institutional Review Board.

\section{Cytometry by time-of-flight}

TILs, NILs and PBMCs from 23 patients with HCC (online supplementary table S1) were analysed by CyTOF, with or without 5-hour stimulation with phorbol 12-myristate 12-acetate (PMA)/Ionomycin as previously described. ${ }^{16}$ Briefly, the cells were thawed and rested overnight in Roswell Park Memorial Institute media with 10\% human serum and stained with a panel of 35 antibodies (online supplementary table S4) and three anti-CD45-antibody barcodes for simultaneous sample processing as previously described. ${ }^{19}$ The generated files were debarcoded $\left(\mathrm{CD} 45^{+}\right)$, gated based on size and DNA content labelled with a DNA-intercalater (DVS Sciences) and live cells that were negative for cisplatin. The individual files were downsampled to 10 000-live CD45 ${ }^{+}$immune cells and analysed using in-house developed Multidimensional-Automated Reduction and Visualisation software ${ }^{17}$ based on a combination of non-linear dimensionality reduction algorithm (Barnes-Hut SNE) and k-means-clustering algorithm. ${ }^{20}$ The CyTOF data were also independently validated using FlowJo software (V.X.0.7, Tree Star) (onlinesupplementary table S1).

\section{Next-generation sequencing}

TILs were isolated from HBV-related HCCs $(n=3-4)$ and non-viral-related HCCs $(n=3)$ and stained with surface markers: CD45, CD3, CD4, CD8, CD45RO, CD103 (all from Biolegend), and PD-1 (BD Biosciences). regulatory $\mathrm{T}$ cells $\left(\mathrm{T}_{\mathrm{REG}}\right)\left(\mathrm{CD} 4^{+} \mathrm{CD} 25^{+} \mathrm{CD} 127^{-}\right)$and resident memory $\mathrm{T}$ cells $\left(\mathrm{T}_{\mathrm{RM}}\right.$; $\mathrm{CD} 8{ }^{+} \mathrm{CD} 103^{+} \mathrm{CD} 45 \mathrm{RO}+$ ) were sorted using a FACSAria-III (BD Biosciences) with 95\%-99\% purity. Total RNA was isolated using a Picopure RNA-Isolation kit (Arcuturus, Ambion) and cDNA was generated using SMART Seqv4 UltraLow Input RNA Kit (Clontech), both according to manufacturer's instructions. Illumina-ready libraries were prepared from cDNA using the Illumina-NexteraXT DNA-Library-Prep Kit (Illumina). Sequencing was performed at the NGS Platform of Genome Institute of Singapore on a HiSeq 2500 platform using HighOutput V.3.

Raw sequencing reads were mapped via Hierarchical Indexing for Spliced Alignment of Transcripts with reference to the Human-Assembly-GRCh38.p7 from Ensembl. Aligned reads were then sorted using SAMtools and the raw gene counts were extracted with high-throughput sequencing data. ${ }^{21}$ The edgeR tool was used to analyse the differential gene expression between two sample groups. The empirical Bayes quasi-likelihood F-test was used in the Generalised Linear Model pipeline for gene-wise statistical analyses. Genes with a fold-change $>2$ and $\mathrm{p}<0.01$ were selected. The data were then visualised in heat maps using R-Script and further annotated with Reactome Pathway Analysis.

\section{In vitro T-cell suppression assay}

The T-cell suppression assay was performed according to a previously published method that used small numbers of tissue-isolated $\mathrm{T}_{\mathrm{REG}}{ }^{22}$ Briefly, TILs were stained with the following anti-human antibodies: CD45, CD3, CD4, CD8, CD25, CD45RO, CD103, CD127 (all from Biolegend) and PD-1 (BD Biosciences). Concurrently, autologous PBMCs were prelabelled with CellTrace Violet dye (C34557, Life Technologies) and then stained with antihuman antibodies: CD45, CD3, CD4, CD25 and CD127. The FACSAria-III (BD Biosciences) was used to isolate PD $-1^{+} \mathrm{T}_{\mathrm{REG}}$ or PD $-1^{-} \mathrm{T}_{\mathrm{REG}}\left(\mathrm{PD}-1^{+} / \mathrm{PD}-1^{-} \mathrm{CD} 45^{+} \mathrm{CD} 3{ }^{+} \mathrm{CD} 4^{+} \mathrm{CD} 25^{+} \mathrm{CD} 127^{\mathrm{lo}}\right.$ cells) 
and $\mathrm{T}_{\mathrm{RM}}$ or non- $\mathrm{T}_{\mathrm{RM}}\left(\mathrm{CD} 103^{+} / \mathrm{CD} 103^{-} \mathrm{CD} 45^{+} \mathrm{CD} 8^{+} \mathrm{CD} 45 \mathrm{RO}^{+-}\right.$ cells) from the TILs and CellTrace Violet-labelled responder $\mathrm{T}$ cells (Tresp; $\mathrm{CD} 45^{+} \mathrm{CD} 3{ }^{+} \mathrm{CD} 4{ }^{+} \mathrm{CD} 25^{-} \mathrm{CD} 127^{\text {hi }}$ cells) from the autologous PBMCs $(n=7 \mathrm{HBV}$-related HCCs from online supplementary table S1). Tresp cells were cocultured in 96-well plates for 5 days with either PD- $1^{+} \mathrm{T}_{\mathrm{REG}}, \mathrm{PD}-1 \mathrm{~T}_{\mathrm{REG}}, \mathrm{T}_{\mathrm{RM}}$ or non- $\mathrm{T}_{\mathrm{RM}}$ at a $1: 1$ ratio (20000 cells each), with or without 20000 Human $T_{\text {REG }}$ Suppression Inspector beads (Miltenyi Biotec). In a separate experiment, the suppression assay was performed with antihuman PD-1 or programmed death-ligand 1 (PD-L1) antibodies with their respective isotype control antibody (all from Biolegend). At the end of these experiments, the cells were harvested and the signal from the CellTrace Violet dye was analysed using a BD LSR FORTESSA with five lasers (BD Biosciences). Data analysis was performed using FlowJo software.

\section{Multiplex tissue IF}

Multiplex tissue IF was performed using an OpalMultiplex Immunohistochemistry system and images were acquired on a Vectra 3.0 Automated Quantitative Pathology Imaging System (Perkin Elmer), as previously described. ${ }^{23}$ 4',6-Diamidino-2-phenylindole was used as the nuclear marker, and the antibodies used are listed in online supplementary table S5. Quantification of positively stained cells was performed using the Vectra 3.0 System and verified by manual counting of 5-10 random fields at $100 \times$ magnification. The mean number from all fields from each patient sample was used to calculate the cellular density (number $/ \mathrm{mm}^{2}$ ).

\section{Statistical analysis}

A Mann-Whitney U test was used in Forest Plot for analysing differential nodes between two groups of clusters. The edgeR package $^{24}$ was used for differential expression analysis of the RNA-seq data. FlowJo data analyses were performed using the Mann-Whitney U test or paired Student's t-test, both two-sided and two-way analysis of variance test with Tukey's multiple comparison post-tests (GraphPad Prism V.6.0f). Univariate and multivariate analyses were performed using Cox proportional hazards models.

\section{RESULTS \\ HBV-related HCC and non-viral-related HCC are composed of distinct immune subsets}

Based on the hypothesis that chronic HBV infection would have a significant impact on TME of HCC, we first aimed to determine whether the TMEs of HBV-related HCC and non-viral-related HCC are distinct. Here, we used CyTOF to perform an in-depth immunoprofiling of the immune landscapes of both HCC aetiologies. Our CyTOF panel consisted of 35 surface and intracellular immune markers (online supplementary table S4) that could examine the global immune phenotypes of TILs, NILs and PBMCs from HBV-related $(n=12)$ and non-viral-related $(n=11)$ HCC tumours (figure 1A and online supplementary table S1). The distributions of the immune lineages from HBV-related HCC versus non-viral-related HCC TILs (including $\mathrm{CD}^{+} \mathrm{T}$ cells, CD4 ${ }^{+} \mathrm{T}$ cells, CD56 ${ }^{+}$natural killer (NK) cells, $\mathrm{CD} 3{ }^{+} \mathrm{CD} 56^{+} \mathrm{NKT}$ cells and $\mathrm{CD} 14^{+}$tumour-associated macrophages (TAM)) were presented as two-dimensional (2D) t-SNE plots (figure $1 \mathrm{~B}$ ). The percentages of each immune lineage were manually gated using FlowJo in each individual TIL, NIL and PBMC, which validated the enrichment of $\mathrm{CD}^{+}{ }^{+} \mathrm{T}$ cells in TILs from HBV-related HCC (figure 1C). Of note, the other major lineages and the TIL density showed no significant differences between the two HCC subtypes (data not shown).

Next, we performed dimensional reduction and clustering of our CyTOF data to generate nodes (immune subsets) with similar immune-marker expression profiles, as previously described. ${ }^{16} \mathrm{~A}$ total of 456 nodes were produced, and after applying MannWhitney U test, we found that 389 nodes $(85.3 \%)$ were shared between the two HCC subgroups and $42(9.2 \%)$ and $25(5.5 \%)$ nodes were enriched in HBV-related HCC and non-viral-related HCC, respectively (figure 1D). These data indicate that the general immune landscapes of HBV-related and non-viral-related HCC are very similar, with $<10 \%$ difference in the immune populations enriched in each HCC subtype.

\section{$T_{R E G}$ and $T_{R M}$ cells are enriched in HBV-related HCC, and Tim- $3^{+} \mathrm{CD}^{+} \mathrm{T}$ cells and CD244 ${ }^{+} \mathrm{NK}$ cells are enriched in non-viral- related $\mathrm{HCC}$}

We further interrogated the frequency of differentially expressed nodes in HBV-related and non-viral-related HCC (figure 2A) and generated a $2 \mathrm{D}$ heat map of the marker expression profiles for these differentially expressed nodes (figure 2B). Here, we observed overall higher expression of multiple pro-inflammatory markers in non-viral-related HCC, namely interferon (IFN)- $\gamma$, interleukin (IL)-17a, granzyme B and tumour necrosis factor (TNF)- $\alpha$ (figure 2B). This observation is consistent with the hypothesis that chronic HBV infection leads to an immunosuppressive microenvironment. $^{25}$

We also noted distinct subsets that could differentiate HBV-related HCC TILs from non-viral-related HCC TILs. These subsets included $\mathrm{T}_{\mathrm{REG}}\left(\mathrm{CD} 4{ }^{+} \mathrm{CTLA}-4^{+} \mathrm{Foxp}^{+}\right)$and $\mathrm{T}_{\mathrm{RM}}\left(\mathrm{CD} 8{ }^{+} \mathrm{CD}_{103}{ }^{+} \mathrm{CD} 45 \mathrm{RO}^{+} \mathrm{CD} 69^{+}\right)$that were enriched in the HBV-related HCC TILs and Tim $-3^{+} \mathrm{CD} 8^{+} \mathrm{T}$ cells and $\mathrm{CD} 244^{+} \mathrm{CD} 56{ }^{+} \mathrm{NK}$ cells that were enriched in the non-viral-related HCC TILs (figure $2 \mathrm{~B}$ and online supplementary figure $\mathrm{S} 1 \mathrm{~A}-\mathrm{C})$. Other immune subsets, such as $\mathrm{CD} 4^{+} \mathrm{T}$ cells, $\mathrm{CD}^{+} \mathrm{T}$ cells and CD14 ${ }^{+}$TAM showed only subtle differences between the two groups (figure 2B). Manual gating using FlowJo (online supplementary figure S2A-C) confirmed that $T_{\text {REG }}$ and $T_{R M}$ were indeed enriched in the TILs from HBV-related HCC (figure 2C). Further analyses showed that $\mathrm{T}_{\text {REG }}$ were specifically enriched in TILs compared with NILs and PBMCs, and $\mathrm{T}_{\mathrm{RM}}$ were enriched in TILs compared with PBMCs in HBV-related HCC (figure 2C). Conversely, Tim $-3{ }^{+} \mathrm{CD} 8{ }^{+} \mathrm{T}$ cells and $\mathrm{CD} 244^{+} \mathrm{CD} 56{ }^{+} \mathrm{NK}$ cells were enriched in the non-viral-related TILs (figure 2C). Taken together, the immune landscape of HBV-related HCC is distinct from non-viral-related HCC with the enrichment of $\mathrm{T}_{\mathrm{REG}}$ and $\mathrm{T}_{\mathrm{RM}}$.

\section{TILs from HBV-related HCC express more PD-1}

As shown in our CyTOF data, the TME of HBV-related HCC was distinct from that of the non-virus-related HCC. We further investigated immune marker expression in TILs between the two groups (online supplementary figure S3A-E), and specifically compared the expression of different costimulatory and coinhibitory molecules (online supplementary figure S3E). Here, we found marked differences in OX40 expression on $\mathrm{CD}^{+}{ }^{+} \mathrm{T}$ cells and $\mathrm{PD}-1$ expression on both $\mathrm{CD}^{+}{ }^{+} \mathrm{T}$ cells and $\mathrm{CD} 8^{+} \mathrm{T}$ cells (figure $3 \mathrm{~A}$ ). Further interrogation of OX40 and PD-1 expression on $\mathrm{T}_{\mathrm{REG}}$ cells (which were enriched in HBV-TILs) demonstrated that $\mathrm{T}_{\mathrm{REG}}$ from HBV-related HCC indeed expressed more OX40 and PD-1 compared with non-virus-related HCC (figure 3B). 

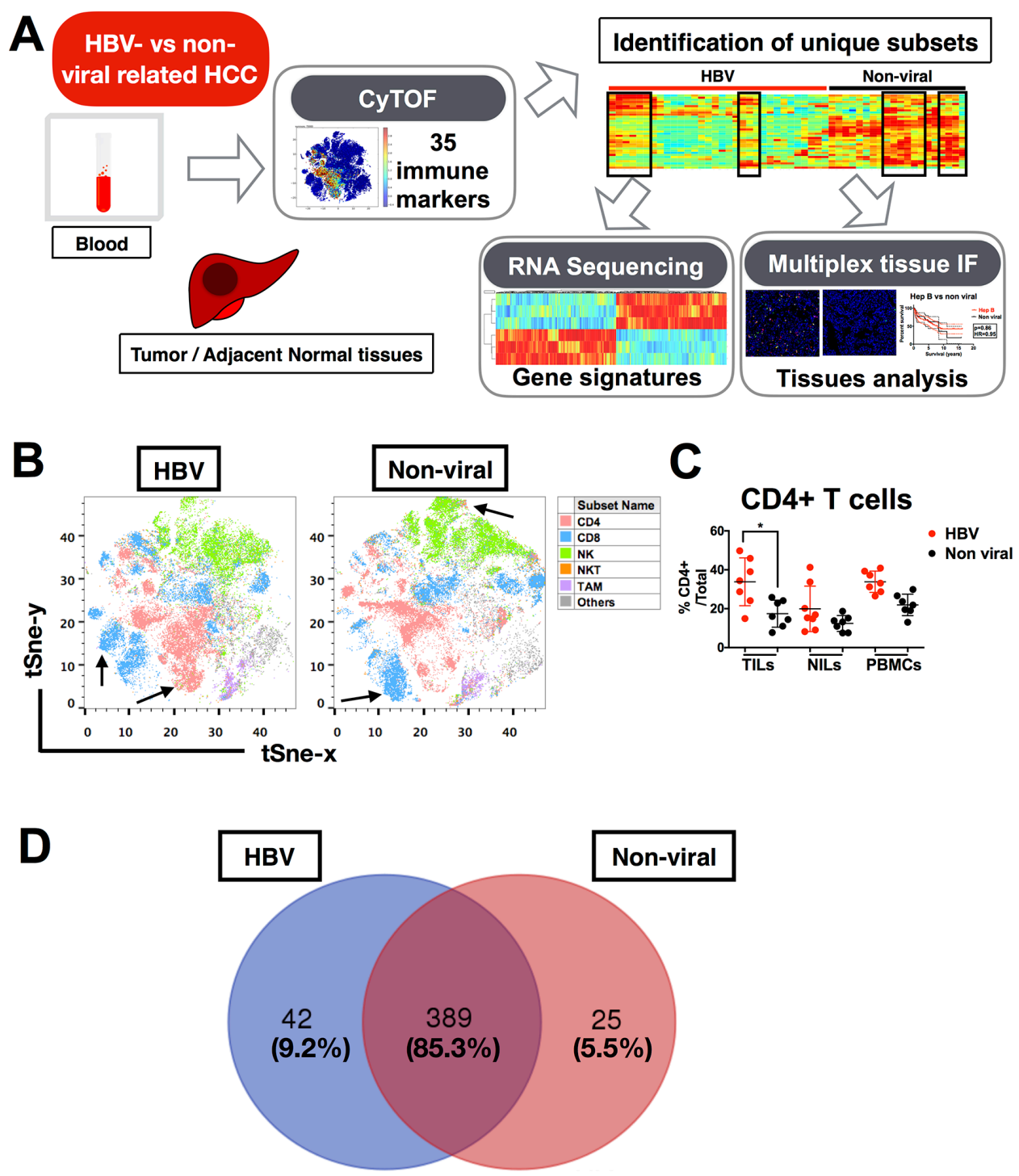

Figure 1 Immune landscape of HBV-related HCC. (A) Study pipeline: TILs, NILs and PBMCs were collected from blood samples and tumour and adjacent tumour tissue from patients with HBV-related HCC and non-viral HCC and were analysed by CyTOF. Unique immune subsets were identified by CyTOF analysis and sorted for further analysis using RNA sequencing. Independent validation was performed using multiplex tissue immunofluorescence on FFPE patient samples. (B) Two-dimensional t-SNE illustration of the CyTOF data of TILs isolated from HBV-related HCC and non-viral-related HCC, gated (with colour coding) on $\mathrm{CD} 4^{+} \mathrm{T}$ cells, CD8 ${ }^{+} \mathrm{T}$ cells, CD56 ${ }^{+} \mathrm{NK}$ cells, CD3 ${ }^{+} \mathrm{CD} 56^{+} \mathrm{NKT}$ cells and CD14 ${ }^{+} \mathrm{HLA}-\mathrm{DR}{ }^{+} \mathrm{TAM}$. Arrows showed enriched clusters in each HCC. (C) Percentages of CD4 ${ }^{+}$T cells from TILs, NILs and PBMCs in HBV-related HCC vs non-viral-related HCC, manually gated using FlowJo software ( $n=7$ for each group). Data represent the means $\pm S D$; * $p<0.05$ (unpaired Student's t-test). (D) Venn diagram showing the number (and percentage) of common and differentially expressed nodes between HBV-related HCCS and non-viral-related HCCs. CyTOF, cytometry by time-of-flight; FFPE, formalin-fixed and paraffin-embedded; HCC, hepatocellular carcinoma; NIL, non-tumour-infiltrating leukocytes; NK, natural killer; PBMC, peripheral blood mononuclear cells; TAM, tumour-associated macrophages; TIL, tumour-infiltrating leukocytes.

Similarly, $\mathrm{T}_{\mathrm{RM}}$ of both TILs and NILs from patients with HBV-related HCC expressed more PD-1 compared with non-virus-related HCC (figure 3C).

We then examined whether higher PD-1 expression on $\mathrm{T}_{\mathrm{RM}}$ from HBV-related HCC corresponded to lower expression of TNF- $\alpha$ and IFN- $\gamma$ on stimulation by PMA/Ionomycin. Indeed, PD-1-expressing $\mathrm{T}_{\mathrm{RM}}$ from HBV-related HCC expressed less TNF- $\alpha$ and IFN- $\gamma$ when compared with that from non-viral-related HCC when stimulated with PMA/ Ionomycin (figure 3D and figure 3E). In general, TILs from HBV-related HCC exhibited higher PD-1 expression and a more exhausted phenotypes than non-viral-related HCC, which is consistent with our hypothesis and previous data that chronic HBV infection induces an immunosuppressive
TME. Despite this finding, the ability of stimulated $T_{R M}$ to express pro-inflammatory cytokines indicated that they remained functionally competent and cytotoxic when appropriately activated intratumourally.

$\mathrm{T}_{\mathrm{REG}}$ and $\mathrm{T}_{\mathrm{RM}}$ isolated from HBV-related or non-viral-related HCC have distinct transcriptomic signatures

As our data showed that $\mathrm{T}_{\mathrm{REG}}$ and $\mathrm{T}_{\mathrm{RM}}$ were enriched in the TME of HBV-related HCC, we decided to examine whether these cells had unique functions or transcriptomic profiles depending on whether they reside in $\mathrm{HBV}$-related or non-viral related HCC. To this aim, we isolated $\mathrm{CD}^{+} \mathrm{CD} 25^{+} \mathrm{CD} 127 \mathrm{~T}_{\mathrm{REG}}$ and $\mathrm{CD}^{+} \mathrm{CD} 103^{+} \mathrm{CD} 45 \mathrm{RO}^{+} \mathrm{T}_{\mathrm{RM}}$ from HBV-related $(\mathrm{n}=3$ or 4$)$ and 

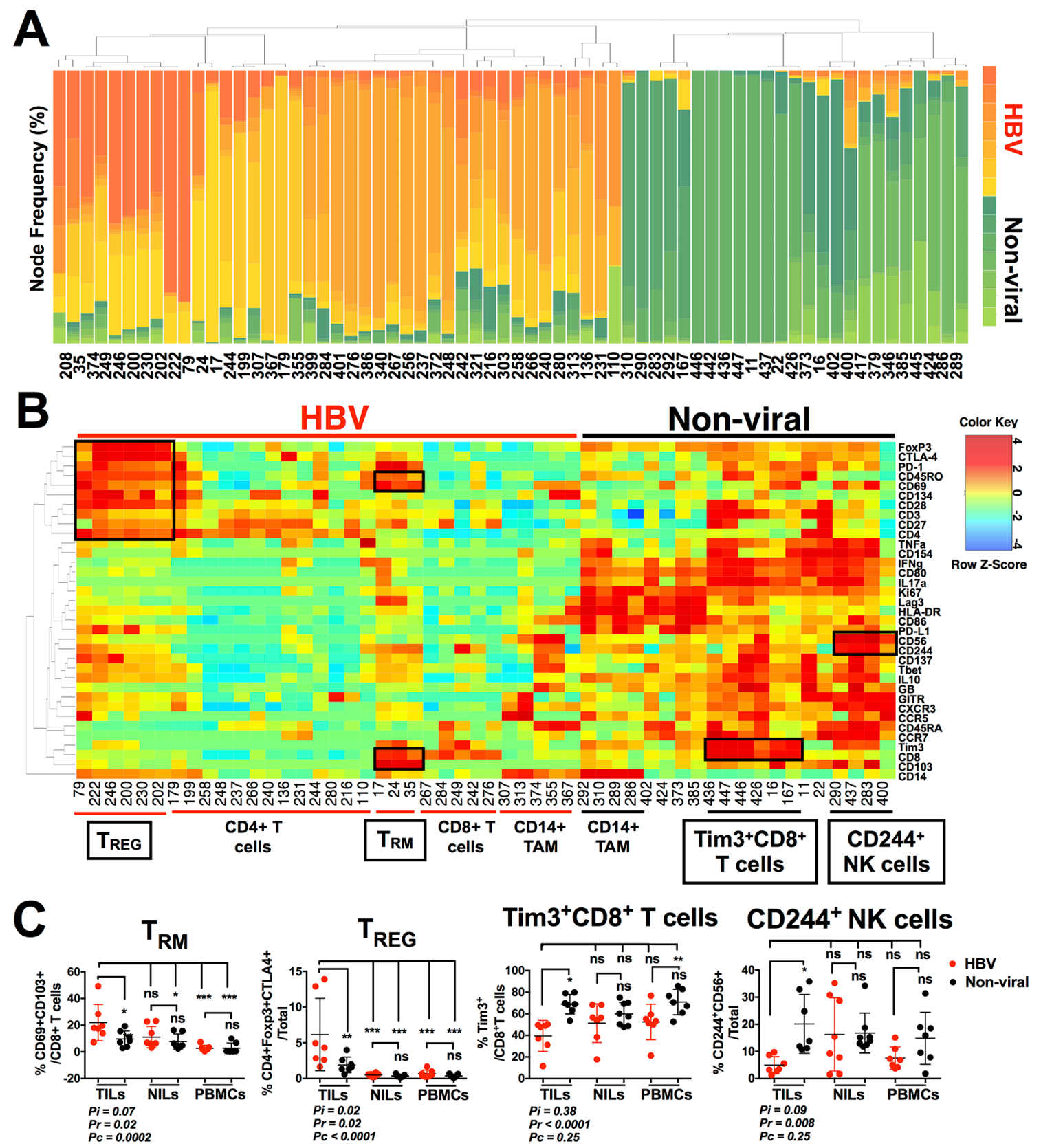

Figure 2 Immune subsets enriched in HBV-related HCC and non-viral-related HCC. (A) Frequency of differentially expressed nodes (immune subsets) in HBV-related HCC vs non-viral-related HCC, identified by unpaired Mann-Whitney U test. Each colour gradient represents a single patient. (B) Twodimensional heat map showing the differential expression of 35 immune markers in HBV-related TILs (red bars) or non-viral-related TILs (black bars). The immune subsets that represent these nodes based on their markers expression are noted. Boxes show the major distinct immune subsets enriched in either of the TILs. (C) Percentages of $\mathrm{T}_{\mathrm{REG}}\left(\mathrm{CD} 4^{+} \mathrm{Foxp3}^{+} \mathrm{CTLA}-4^{+}\right), \mathrm{T}_{\mathrm{RM}}\left(\mathrm{CD} 8^{+} \mathrm{CD} 103^{+} \mathrm{CD} 69^{+}\right)$, Tim $-3^{+} \mathrm{CD} 8^{+} \mathrm{T}_{\text {cells }}$ and CD244 $4^{+} \mathrm{NK}$ cells from TILs, NILs or PBMCs from either HBV-related HCC or non-viral-related HCC as obtained by manual gating using FlowJo software. Data represent the means \pm SD; two-way analysis of variance test with Tukey's multiple comparison post-tests. Pi=interaction p value (interaction between viral status and TILs/NILs/ PBMCs); Pr=row factor (viral status) $p$ value; Pc=column factor (TILs/NILs/PBMCs) $p$ value; ${ }^{*} p<0.05,{ }^{*} p<0.01,{ }^{* * *} p<0.001$. HCC, hepatocellular carcinoma; NIL, non-tumour-infiltrating leukocytes; NK, natural killer; ns, non-significant; PBMC, peripheral blood mononuclear cells; TIL, tumourinfiltrating leukocytes.

non-viral-related $(n=4)$ HCCs and performed NGS on these sorted cells (online supplementary figureS4A). The NGS results showed differential gene signatures of both $T_{R E G}$ and $T_{R M}$ from HBV-related and non-viral-related HCC (figure 4A), with a total of 289 and 166 differentially expressed genes, respectively. We analysed the enriched genes from the $T_{\text {REG }}$ (58 genes) and $T_{R M}$ (111 genes) isolated from HBV-related HCC using the Reactome functional analysis tool (figure 4B, online supplementary table S6 and S7).

$\mathrm{T}_{\mathrm{REG}}$ from HBV-related HCC showed higher expression of FOXP3 (figure 4A) and other genes involved in the IL-10 signalling pathway such as IL1R1 and TNFRSF1B (figure 4B and online supplementary table S6), indicating a more immunosuppressive phenotype of $\mathrm{T}_{\mathrm{REG}}$ isolated from HBV-related 


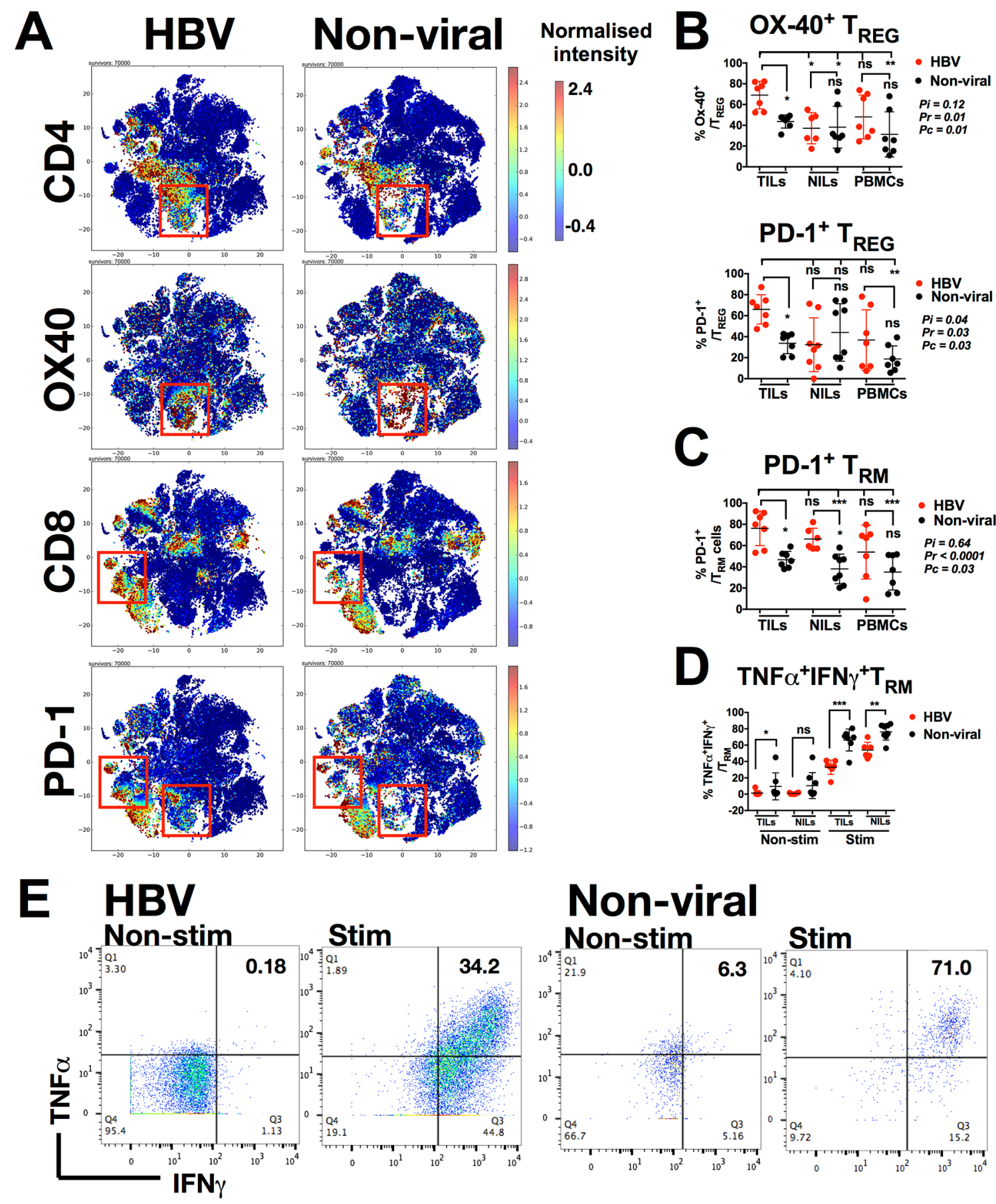

Figure 3 Phenotypes of $\mathrm{T}_{R E G}$ and $\mathrm{T}_{\mathrm{RM}}$ enriched in HBV-related HCC. (A) Two-dimensional cellular t-SNE plots from the CyTOF data showing differentially expressed markers for TILs isolated from HBV-related HCC vs non-viral-related HCC. The red boxes indicate nodes enriched in either HBVrelated HCCS or non-viral-related HCCS, respectively. (B) Percentage of $0 x-40^{+}$and PD- $1^{+} T_{R E G^{*}}$ (C) Percentage of PD- $1^{+} T_{R M}$ (D) Percentage of TNF- $\alpha+$ IFN- $\gamma+T_{\text {RM }}$ non-stimulated (Non-stim) or stimulated (Stim) with PMA/lonomycin. (E) Representative plots showing expression of TNF- $\alpha$ and IFN- $\gamma$ on $\mathrm{T}_{\mathrm{RM}}$ from HBV-related or non-viral-related HCC non-stimulated (Non-stim) or stimulated (Stim) with PMA/lonomycin. B-D: Data were obtained by manual gating using FlowJo software and each graph represent the means \pm SD; B and C: two-way analysis of variance test with Tukey's multiple comparison post-tests. $\mathrm{Pi}=$ interaction $\mathrm{p}$ value (interaction between viral status and TILs/NILs/PBMCs); $\mathrm{Pr}=$ row factor (viral status) $\mathrm{p}$ value; $\mathrm{Pc}=$ column factor (TILs/NILs/PBMCs) p value; D: unpaired Student's t-test. ${ }^{*} \mathrm{p}<0.05,{ }^{* *} \mathrm{p}<0.01,{ }^{* * *} \mathrm{p}<0.001$. CyTOF, cytometry by time-of-flight; HCC, hepatocellular carcinoma; IFN, interferon; NIL, non-tumour-infiltrating leukocytes; ns, non-significant; PBMC, peripheral blood mononuclear cells; PD1, programmed cell death protein 1; TIL, tumour-infiltrating leukocytes; TNF, tumour necrosis factor.

HCC compared with $\mathrm{T}_{\mathrm{REG}}$ from non-virus-related HCC. IL-10 signalling has also been shown to sustain FOXP3 expression, thereby stabilising $\mathrm{T}_{\mathrm{REG}}$ 's phenotypes and functions. ${ }^{26} L A G 3$, an exhaustion-related gene was also enriched in $\mathrm{T}_{\mathrm{REG}}$ from HBV-related HCC (figure 4A) and it was previously reported to be important for its maximal suppressive activity. ${ }^{27}$ Furthermore, it was reported that LAG-3 expressing $T_{\text {REG }}$ were enriched in the tumour microenvironment and were immunosuppressive based on their high expression of IL-10 and transforming growth factor (TGF)- $\beta .^{28}$ The notable upregulation of cell cycle-related 
A

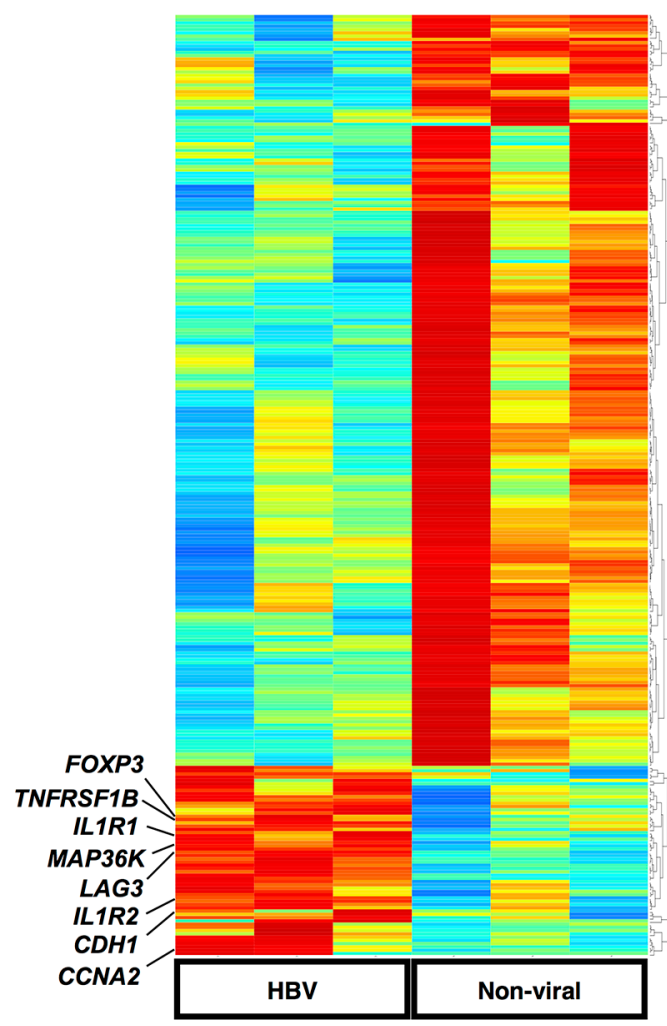

B

Functional annotation of enriched genes in TREG from HBV-related HCC
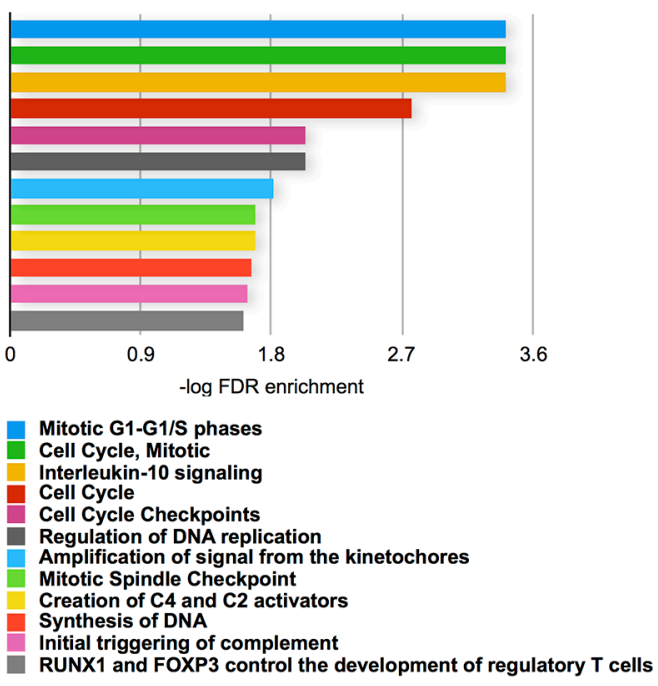

TREG

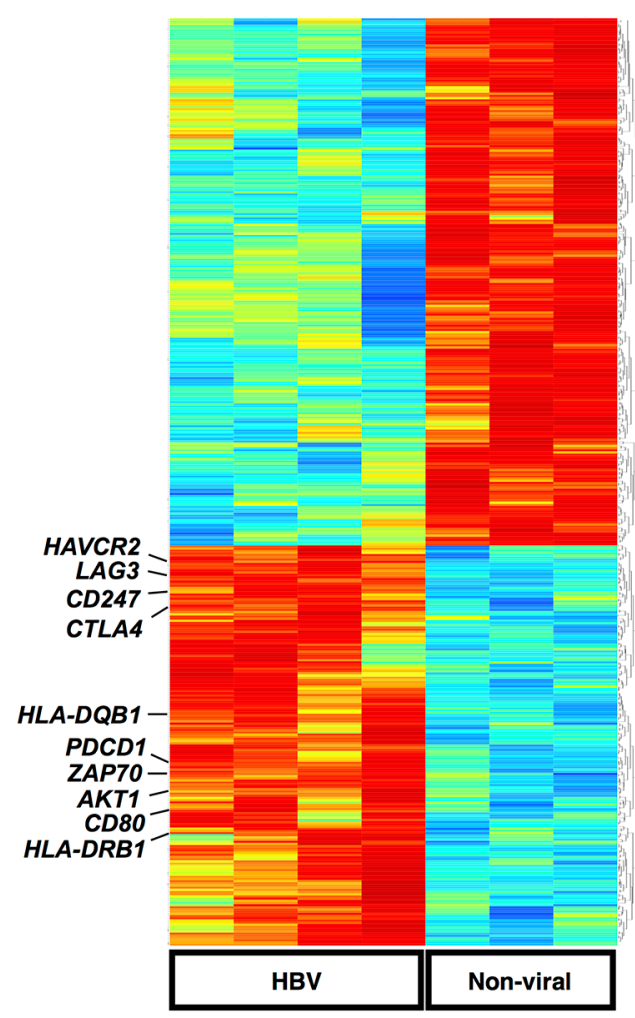

Color Key

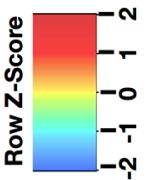

Functional annotation of enriched genes in TRM from HBV-related HCC

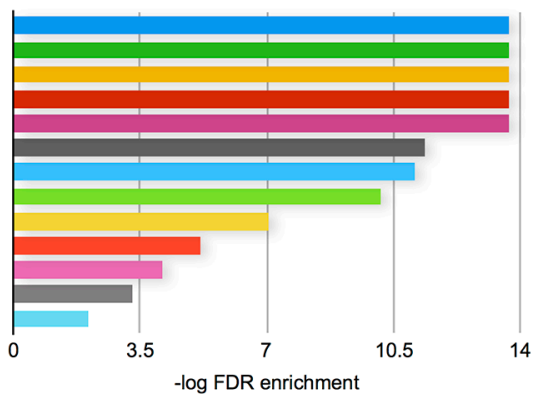

Translocation of ZAP-70 to Immunological synapse PD-1 signaling

Phosphorylation of CD3 and TCR zeta chains

Costimulation by the CD28 family

Generation of second messenger molecules

Downstream TCR signaling

TCR signaling

MHC class II antigen presentation

Interferon gamma signaling

Interferon Signaling

Cytokine Signaling in Immune system

Adaptive Immune System

Immune System

Figure 4 Transcriptome analysis of $\mathrm{T}_{\mathrm{REG}}$ and $\mathrm{T}_{\mathrm{RM}}$ isolated from HBV-related HCC vs non-viral-related HCC. (A) Differentially expressed genes identified by NGS analysis on $\mathrm{T}_{\mathrm{REG}}$ and $\mathrm{T}_{\mathrm{RM}}$ isolated from HBV-related HCC vs non-viral-related HCC. (B) Reactome pathway analysis of the enriched genes identified from $\mathrm{T}_{\mathrm{REG}}$ and $\mathrm{T}_{\mathrm{RM}}$ isolated from HBV-related HCCs compared with non-viral-related HCC. HCC, hepatocellular carcinoma; NGS, nextgeneration sequencing.

pathways (figure 4B) suggests that these $T_{\text {REG }}$ were proliferative. Taken together, these findings were consistent with our earlier data that demonstrated a more immunosuppressive phenotype of $\mathrm{T}_{\mathrm{REG}}$ from HBV-related HCC compared with non-viral-related HCC.

Genes enriched in $\mathrm{T}_{\mathrm{RM}}$ from HBV-related HCC exhibited a state of exhaustion, as implicated by the higher expression of exhaustion marker genes such as PDCD1 (PD-1), LAG3, HAVCR2 (Tim-3) and CTLA4 (figure 4A and online supplementary table S7). These data were again consistent with our CyTOF analysis and support our hypothesis of an exhausted TME in HBV-related HCC. Other common pathways related to genes enriched in $\mathrm{T}_{\mathrm{RM}}$ from HBV-related HCC included IFN signalling, cytokine signalling and adaptive immune system which may 

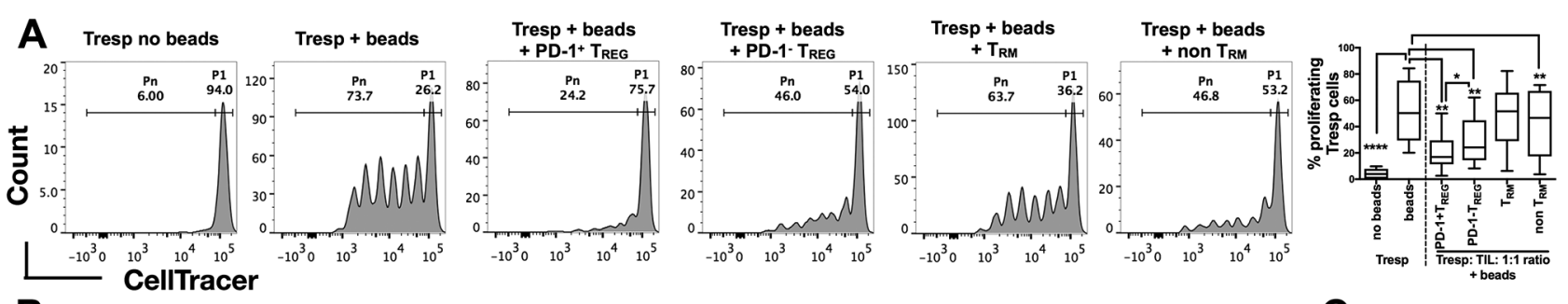

B
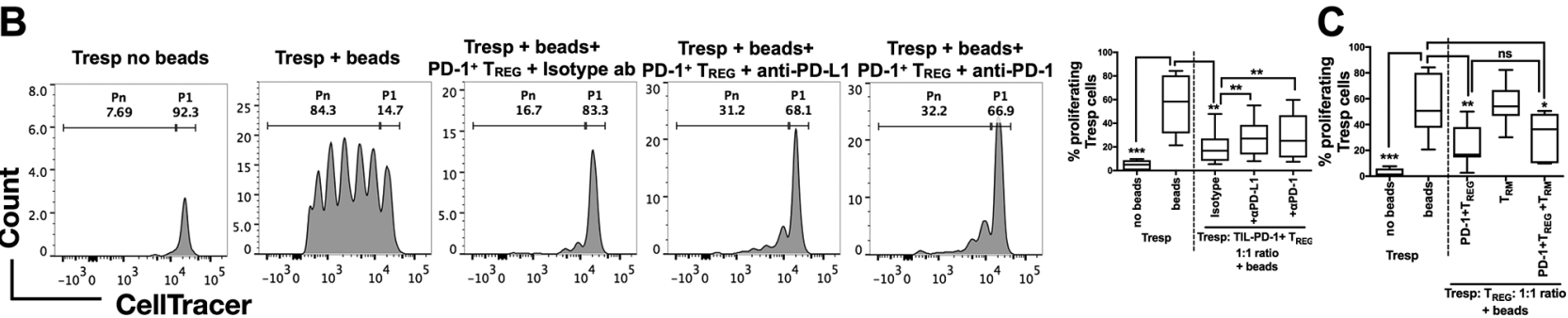

Figure 5 Suppressive functions of $\mathrm{T}_{\mathrm{REG}}$ isolated from HBV-related HCC. (A) Representative analysis of CellTrace Violet-labelled Tresp cells with or without human $\mathrm{T}_{\mathrm{REG}}$ suppression inspector beads (upper panel) and with beads as well as different immune subsets isolated from TILs (PD- ${ }^{+} /$

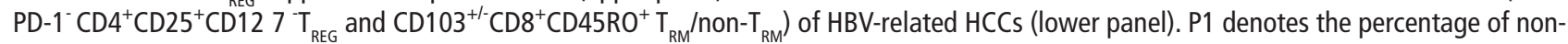
proliferating Tresp and Pn denotes the percentage of proliferating Tresp. The percentages of proliferating Tresp cells under different coculture conditions are shown in the graph on the right ( $n=7$ replicates in each condition). (B) Representative analysis of CellTrace Violet-labelled Tresp cells with or without beads (upper panel) and with beads, PD- $1^{+} \mathrm{T}_{\mathrm{REG}}$ and anti-human PD-1, anti-human PD-L1 or control isotype antibody (lower panel). P1 denotes non-proliferating Tresp and Pn denotes proliferating Tresp. The percentages of proliferating Tresp cells under different coculture conditions with or without checkpoint blockade are shown in the graph on the right ( $n=7$ replicates in each condition). (C) Percentages of proliferating Tresp cells when cocultured with PD- $1^{+} T_{R E G} T_{R M}$ or both TILs combined ( $n=7$ replicates in each condition). A-C: Paired student's t-test. ${ }^{*} p<0.05 ;{ }^{* *} p<0.01$; ${ }^{* * *} \mathrm{p}<0.001 ;{ }^{* * *} \mathrm{p}<0.0001$. HCC, hepatocellular carcinoma; ns, non-significant; PD-1, programmed cell death protein 1; PD-L1, programmed deathligand 1; TIL, tumour-infiltrating leukocytes

indicate a prior activation leading to exhaustion in these cells (figure 4B). In conclusion, our transcriptome analysis of $\mathrm{T}_{\mathrm{REG}}$ and $\mathrm{T}_{\mathrm{RM}}$ further validated that the TME of HBV-related HCC is more immunosuppressive/exhausted than non-viral HCC.

\section{$P D-1^{\text {hi }} T_{R E G}$ is more immunosuppressive and their phenotype can be diminished on PD-1/PD-L1 blockade}

We next investigated the functions of $T_{R E G}$ and $T_{R M}$ from HBV-related HCC using an in vitro T-cell suppression assay. ${ }^{22}$ As HBV-related HCC was enriched with PD- $1^{+} \mathrm{T}_{\mathrm{REG}}$ (figure $3 \mathrm{~B}$ ), we first aimed to address whether PD- $1^{+} \mathrm{T}_{\mathrm{REG}}$ and PD-1- $\mathrm{T}_{\mathrm{REG}}$ are functionally distinct. We isolated $\mathrm{PD}-1^{+} \mathrm{T}_{\mathrm{REG}}$ and $\mathrm{PD}-1^{-} \mathrm{T}_{\mathrm{REG}}$ together with $\mathrm{T}_{\mathrm{RM}}$ and non- $\mathrm{T}_{\mathrm{RM}}$ from HBV-related HCC tumours to examine their ability to suppress the proliferation of $\mathrm{CD} 4{ }^{+} \mathrm{C}$ D $127^{+} \mathrm{CD} 25^{-}$Tresp cells isolated from autologous PBMCs (online supplementary figure S5A and figure S5B). Indeed, we observed significant suppression of Tresp cell proliferation by both PD- $1^{+} \mathrm{T}_{\mathrm{REG}}$ and PD- $1^{-} \mathrm{T}_{\mathrm{REG}}$, with PD $-1^{+} \mathrm{T}_{\mathrm{REG}}$ being significantly more suppressive than its PD-1- counterpart (figure $5 \mathrm{~A}$ and online supplementary figure S6A) despite the fact that they express comparable level of Foxp3 (supplementary figure S5A). $\mathrm{T}_{\mathrm{RM}}$ had no significant effect on suppressing proliferation of Tresp cells (figure 5A) indicating that despite their exhausted phenotypes they did not have immunosuppression function. To further interrogate whether suppression of T-cell proliferation mediated by PD-1 ${ }^{+} \mathrm{T}_{\mathrm{REG}}$ cells could be blocked, we repeated the experiments and incubated the PD $-1^{+} \mathrm{T}_{\mathrm{REG}}$ with either antiPD-1 or anti-PD-L1 antibodies. The suppressive effect of PD-1 ${ }^{+}$ $\mathrm{T}_{\mathrm{REG}}$ was significantly reduced on exposure to either anti-PD-1 or anti-PD-L1 antibodies (figure 5B and online supplementary figure $\mathrm{S6B}$ ) compared with control isotype antibody. Of note, our CyTOF data showed that PD $-1^{+} \mathrm{T}_{\mathrm{REG}}$ also expressed PD-L1 (online supplementary figure S5C). In addition, we found that the suppressive phenotype of $\mathrm{T}_{\mathrm{REG}}$ remained unaffected by coculturing with $\mathrm{T}_{\mathrm{RM}}$, another HBV-TIL-enriched subset (figure 5C).

These functional data demonstrate that the TME of HBV-related HCC, which is enriched with $\mathrm{PD}-1^{+} \mathrm{T}_{\mathrm{REG}}$ (figure $3 \mathrm{~B}$ ), could potentially be more immunosuppressive than the TME of non-virus-related HCC. This immunosuppression could, however, be reversed on application of anti-PD-1/anti-PD-L1 blocking antibodies.

\section{Tumour-infiltrating $\mathrm{T}_{\mathrm{REG}}$ and $\mathrm{T}_{\mathrm{RM}}$ have opposing effects on the} overall survival in patients with HCC

Our data thus far have indicated that $\mathrm{T}_{\mathrm{REG}}$ and $\mathrm{T}_{\mathrm{RM}}$ are the two major immune subsets enriched in HBV-related HCC. We then investigated their contribution to the overall survival (OS) of patients with HCC. Using multiplexed tissue IF on TMA cohort $(n=102)$ (online supplementary table S2), we first validated that both $\mathrm{T}_{\mathrm{REG}}\left(\mathrm{CD} 4^{+} \mathrm{Foxp}^{+}\right)$and $\mathrm{T}_{\mathrm{RM}}\left(\mathrm{CD} 8^{+} \mathrm{CD} 103^{+}\right)$were enriched in HBV-related HCC compared with non-viral-related HCC (figure 6A). Next, we segregated the patients into high or low $\mathrm{T}_{\mathrm{REG}}$ or $\mathrm{T}_{\mathrm{RM}}$ according to median number of cells $/ \mathrm{mm}^{2}$ and examined their OS profiles by Kaplan-Meier analysis. Interestingly, we found that these two immune-subsets had an opposing influence on OS: higher numbers of tumour-infiltrating $\mathrm{T}_{\mathrm{REG}}$ were associated with a poorer survival profile, whereas a higher number of tumour-infiltrating $\mathrm{T}_{\mathrm{RM}}$ was associated with a better survival profile (figure 6B). These findings are consistent with previous studies that showed tumour infiltration of $\mathrm{T}_{\mathrm{REG}}$ is associated with poor prognosis in HCC. ${ }^{29}$ However, the association of $\mathrm{T}_{\mathrm{RM}}$ to a better prognosis of HCC was an interesting and novel observation. Due to the opposing (balancing) effects of $\mathrm{T}_{\mathrm{REG}}$ and $\mathrm{T}_{\mathrm{RM}}$, we would expect the $\mathrm{OS}$ of patients with HBV-related HCC to be equivalent to those with non-viral-related HCC. Indeed, when we compared the OS of patients with HBV-related HCC with those with non-viral-related HCC 

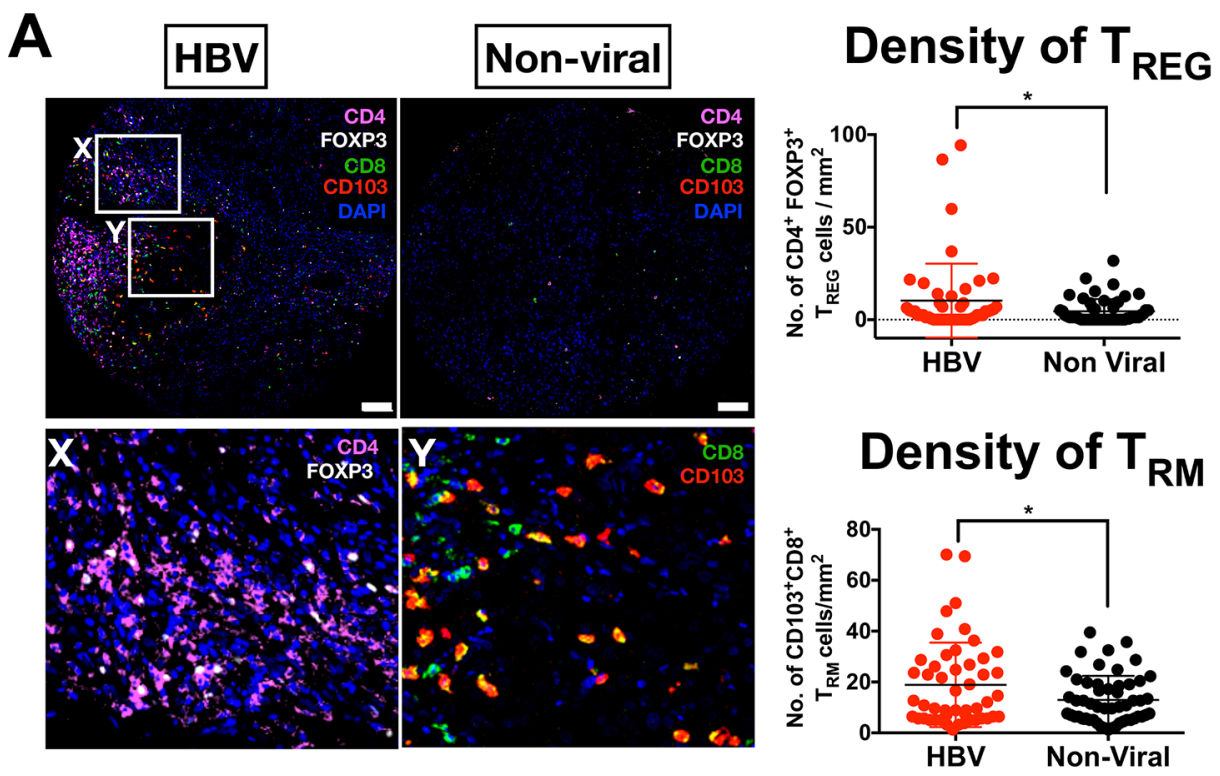

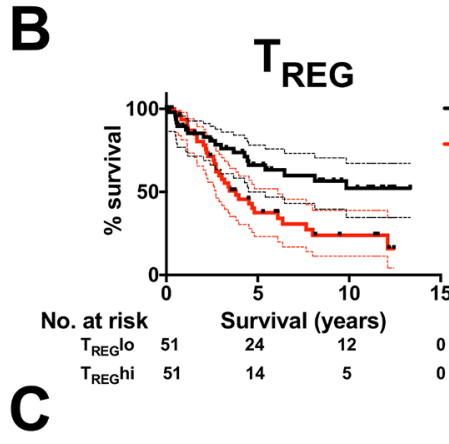

HBV vs non-viral

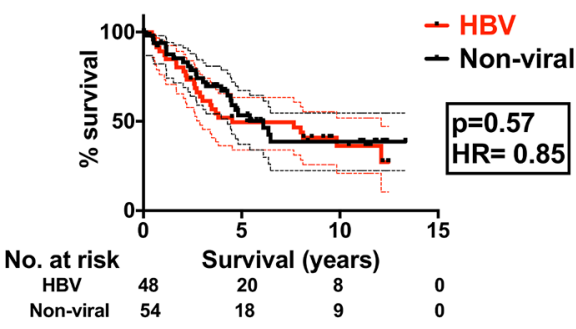

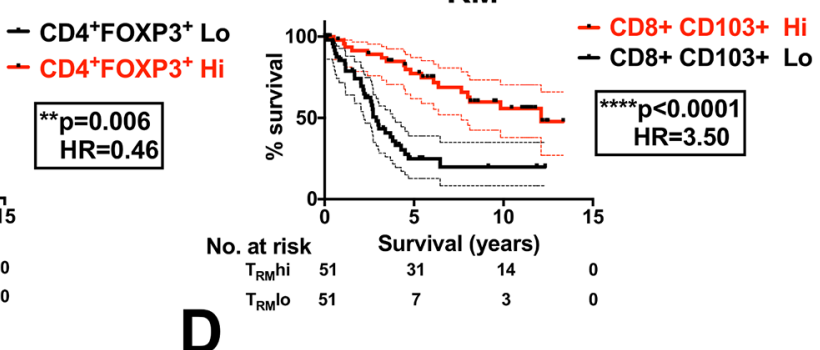

HBV vs non-viral

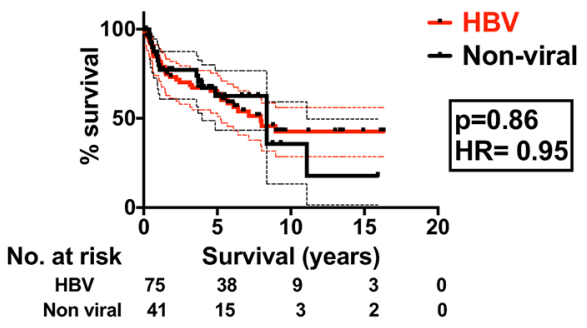

Figure 6

Figure $6 \mathrm{~T}_{\mathrm{REG}}$ and $\mathrm{T}_{\mathrm{RM}}$ enrichment in HBV-related HCC impacts on patient survival. (A) Multiplex immunofluorescence staining of FFPE HCC tissues microarray $(n=102)$ showing enriched $\mathrm{T}_{\mathrm{REG}}\left(\mathrm{CD} 4^{+} \mathrm{Foxp}^{+}\right)$and $\mathrm{T}_{\mathrm{RM}}\left(\mathrm{CD}^{+} \mathrm{CD} 103^{+}\right)$in tumour tissues from HBV-related HCC vs non-viral-related HCC. Magnification, 10x; scale bar, $50 \mu \mathrm{m}$. The lower images show the highlighted boxes $(X, Y)$ at 20x zoom-in, with Foxp3 and CD4 (for $\left.T_{R E G}\right)$ and CD103 and CD8 (for $T_{R M}$ ) shown separately. The densities of $T_{R E G}$ or $T_{R M}$ (no. of cells $/ \mathrm{mm}^{2}$ ) were shown in the graphs on the right. * $p<0.05$. (B) Kaplan-Meier analysis showing the overall patient survival profiles based on high (Hi) or low (Lo) $T_{R E G}$ or $T_{R M}$. The cut-off was based on the median density of $T_{\text {REG }}$ and $\mathrm{T}_{\mathrm{RM}^{\prime}}$ respectively (log-rank test). (C) Kaplan-Meier survival analysis based on viral status in patients with HCC included in the tissue microarray cohort $(n=102)$. (D) Kaplain-Meier survival analysis based on viral status in independent HCC cohort from Singapore, Hong Kong and Zurich $(n=112)$. DAPI, 4',6-D iamidino-2-phenylindole; FFPE, formalin-fixed and paraffin-embedded; HCC, hepatocellular carcinoma.

from the same TMA cohort $(n=102)$, we observed no significant differences (figure 6C). These data were further validated, by analysing the OS of the international HCC cohort from Singapore, Hong Kong and Zurich $(n=116$; online supplementary table S3 and figure 6D). Furthermore, we performed both univariate and multivariate analyses along with several clinical parameters, and demonstrated that intratumoural $\mathrm{T}_{\mathrm{REG}}$ and $\mathrm{T}_{\mathrm{RM}}$ densities were independent and superior prognostic factors for OS in these patients with HCC (table 1). This in-depth analysis of the major immune subsets in HBV-related HCC has identified a complex relationship between immune cell enrichment and the OS profile of patients with HCC.

\section{DISCUSSION}

Several studies have interrogated the immune landscape of HBV-related HCC, ${ }^{34}$ but most have exclusively focused on a single immune subset or on soley HBV-related HCC without a cross-comparison to non-viral-related HCC. These studies thus provide only a restricted view of the immune landscapes 
Table 1 Univariate and multivariate analysis with Cox proportional hazard regression model (TMA cohort)

\begin{tabular}{|c|c|c|c|c|}
\hline \multirow[b]{2}{*}{ Variables } & \multicolumn{2}{|c|}{ Univariate analysis } & \multicolumn{2}{|c|}{ Multivariate analysis } \\
\hline & $\mathrm{HR}(95 \% \mathrm{Cl})$ & $P$ values & HR $(95 \% \mathrm{Cl})$ & $P$ values \\
\hline$T_{\mathrm{REG}}$ density (no./mm²) (<median vs $\geq$ median) & $2.4(1.4$ to 4.1$)$ & 0.001 & 3.6 (2.0 to 6.6$)$ & $2.9 \mathrm{E}-05$ \\
\hline$T_{\mathrm{RM}}$ density (no./mm²) (<median vs $\geq$ median) & $0.3(0.2$ to 0.5$)$ & $1.7 \mathrm{E}-06$ & $0.2(0.1$ to 0.4$)$ & $6.0 \mathrm{E}-07$ \\
\hline TNM stage (I/II/IIII/IV) & $1.5(1.2$ to 2.1$)$ & 0.004 & $1.1(0.8$ to 1.6$)$ & 0.597 \\
\hline Grade $(1 / 2 / 3 / 4)$ & $1.3(0.9$ to 1.8$)$ & 0.150 & $1.1(0.8$ to 1.6$)$ & 0.648 \\
\hline Tumour size $(\mathrm{cm})$ (<median vs $\geq$ median) & 1.6 (1.0 to 2.7$)$ & 0.068 & $1.5(0.9$ to 2.6$)$ & 0.170 \\
\hline AFP level $(\mu \mathrm{g} / \mathrm{mL})(<$ median vs $\geq$ median $)$ & 1.7 (1.0 to 2.8$)$ & 0.045 & 1.8 (1.0 to 3.2$)$ & 0.056 \\
\hline
\end{tabular}

AFP, alpha-fetoprotein; TMA, tissue-microarray; TNM, tumour, node, metastases.

of HCC; a broader understanding is critical to predicting clinical outcomes and designing effective disease-management strategy. Our current study provides a comprehensive examination of the intratumoural immune landscapes of HBV-related HCC compared with non-virally induced HCC, using the high-dimensional analytical tools: CyTOF and NGS. This approach allowed us to address the fundamental impact of the underlying TME on TIL phenotypes. First, we identified the immune subsets that were distinct between the HBV-related and non-viral-related HCC. Specifically, $\mathrm{T}_{\mathrm{REG}}$ and $\mathrm{T}_{\mathrm{RM}}$ were enriched in HBV-related HCC and Tim $-3^{+} \mathrm{CD} 8{ }^{+} \mathrm{T}$ cells and CD $244^{+}$NK cells were enriched in non-viral HCC. We could also clearly visualise a more immunosuppressive or exhausted TME in HBV-related HCC. Given that the $T_{R E G}$ identified in HBV-related HCC expressed higher levels of PD-1, we tested the effects of PD-1/PD-L1 pathway blockade on $\mathrm{T}_{\mathrm{REG}}$ function and found that indeed it could diminish the $T_{\text {REG }}$ suppressive phenotype. Finally, we described the opposing effects of the two HBV-related HCC-enriched immune subsets, $\mathrm{T}_{\mathrm{REG}}$ and $\mathrm{T}_{\mathrm{RM}}$, on the OS in patients with HCC. As expected, those with high $\mathrm{T}_{\text {REG }}$ exhibited worse OS, but interestingly, those with high $\mathrm{T}_{\mathrm{RM}}$, showed more favourable OS.

Our finding of a higher density of intratumoural $T_{\text {REG }}$ in HBV-related HCC is consistent with previous studies that have identified higher $\mathrm{T}_{\mathrm{REG}}$ numbers in chronic HBV infection. ${ }^{30}$ Others have also shown that $\mathrm{T}_{\mathrm{RM}}$ numbers are increased in livers with chronic HBV infection compared with healthy livers. ${ }^{31}$ Recent large-scale single-cell RNA-sequencing on T cells from a majority of HBV-related HCCs also found that $\mathrm{T}_{\mathrm{REG}}$ and exhausted CD $8^{+} \mathrm{T}$ cells (with similar gene signatures as our current study) were enriched in the tumour. ${ }^{32}$ The important roles of NK cells in HBV infection and HCC have been widely described. ${ }^{33}{ }^{34}$ CD244/2B4 is important for effective immune control during chronic viral infections ${ }^{35}$; reduced CD244 on NK cells is associated with increased TGF- $\beta$ during the immune tolerant phase of persistent HBV infection. ${ }^{36}$ The lower percentage of CD $244^{+} \mathrm{NK}$ cells from HBV-related HCC in our study is hence consistent with these previous reports. CD244 also mediates the antitumour activity of NK cells, ${ }^{37}$ which suggests its critical role in non-viral related HCC, that is enriched with $\mathrm{CD} 244^{+} \mathrm{NK}$ cells. On the contrary, Tim-3 is upregulated on multiple immune subsets, including $\mathrm{T}$ cells and NK cells, during chronic HBV infection. ${ }^{38} 39$ The higher Tim $-3^{+} \mathrm{CD}^{+}$levels observed in non-viral HCC in our study thus contrast this general consensus. As many of the previous studies compared the immune profiles of chronic $\mathrm{HBV}$-infected patients with healthy individuals, it is hard to determine whether the same conclusions should have been expected from our study, which compared HCC patients with or without HBV infection.
The immunosuppressive activity of $\mathrm{T}_{\mathrm{REG}}$ and its role in tumour progression has been well documented in many cancers, ${ }^{40}$ including HCC. ${ }^{41} \mathrm{~A}$ higher density of $\mathrm{T}_{\mathrm{REG}}$ cells, intratumourally or in the peripheral blood, is considered a poor prognostic marker for survival of patients with HCC, ${ }^{29}$ as consistent with our current findings. We and others, have also reported CD8 as good prognostic factor in HCC. ${ }^{342} \mathrm{~T}_{\mathrm{RM}}$ was suggested to exert a partial immune response in chronic HBV infection ${ }^{31}$ and was recently reported to be associated with superior survival in other cancers. ${ }^{434}$ Furthermore, the enrichment of CD80 by $\mathrm{T}_{\mathrm{BM}}$ from HBV-related HCC shown in our NGS analysis (figure 4A and online supplementary table S6) and the ability to express cytokines on stimulation (figure 3D) suggests potential T-cell cytotoxicity. As such, our study is the first of its kind uniquely describing the opposing effects of these two immune subsets on the OS of patients with HCC.

Increased expression of PD- 1 on $\mathrm{T}_{\mathrm{REG}}$ and $\mathrm{T}_{\mathrm{RM}}$ in HBV-related HCC is consistent with a virus-induced immunosuppressive/exhausted TME. Our NGS data also supported more immunosuppressive/exhausted phenotypes of $T_{R E G}$ and $T_{R M}$ isolated from HBV-related HCC. As both of these populations expressed PD-1, we would assume that application of PD-1 inhibitor would render a survival benefit. This hypothesis, however, was not supported by the results from the recent CheckMate 040 clinical trial that found no significant difference in response rate for patients with HBV-related or non-viral-related HCC towards nivolumab. ${ }^{14}$ From our current findings, we did observe an alleviated immunosuppressive phenotype when blocking PD-1/PD-L1 pathway on PD $-1^{+} \mathrm{T}_{\mathrm{REG}}$ cells. By contrast, others have reported that blocking PD-1/ PD-L1 pathway would expand the $\mathrm{T}_{\mathrm{REG}}$ population leading to an overall more immunosuppressive phenotype. ${ }^{45}$ The higher number of PD-1-expressing $\mathrm{T}_{\mathrm{RM}}$ cells also does not necessarily translate into an ability to mount an immune response on PD-1 blockade, as some of these $\mathrm{PD}-1^{+} \mathrm{CD} 8^{+} \mathrm{T}$ cells can become terminally exhausted during chronic viral infection. ${ }^{46}$ The effect of PD-1/PD-L1 blockade on multiple immune subsets in HBV-related HCC requires further investigation. Based on the current findings however, we consider immunotherapies which target $\mathrm{T}_{\mathrm{REG}}$ and PD-1-expressing cytotoxic $\mathrm{T}$ cells would be a rational combinatorial strategy for HBV-related HCC. By contrast, immunotherapies that target $\mathrm{NK}$ cells or Tim $-3^{+} \mathrm{CD} 8^{+}$ $\mathrm{T}$ cells may be more effective in non-viral-related HCC.

In conclusion, our study is one of the most comprehensive analyses in immune landscapes of patients with HCC with or without HBV infection. Such deep immunophenotyping strategies are essential in enhancing our understanding of tumour immunity in cancers derived from different aetiologies and will help guide the design of novel immunotherapeutic strategies. 


\section{Author affiliations}

${ }^{1}$ Translational Immunology Institute (TII), SingHealth-DukeNUS Academic Medical Centre, Singapore, Singapore

${ }^{2}$ Department of Pathology, Singapore General Hospital, Singapore, Singapore ${ }^{3}$ Duke-NUS Medical School, Singapore, Singapore

${ }^{4}$ Singapore Immunology Network (SIgN), Agency for Science, Technology and Research (A*STAR), Singapore, Singapore

${ }^{5}$ National Cancer Centre, Singapore, Singapore

${ }^{6}$ Department of Hepatopancreatobiliary and Transplant Surgery, Singapore General Hospital, Singapore, Singapore

${ }^{7}$ Division of Chronic Inflammation and Cancer, German Cancer Research Center (DKFZ), Heidelberg, Germany

${ }^{8}$ Department of Pathology and State Key Laboratory for Liver Research, The University of Hong Kong, Hong Kong, Hong Kong

Acknowledgements The authors would like to thank Fiona Ni Ni Moe and Phang Su Ting from Singapore General Hospital (SGH), Professor Achim Weber from University Hospital of Zurich and Professor Ronnie Poon from Queen Marry Hospital Hong Kong for coordinating the patient sample collection and for assisting with obtaining patient consents. The authors would like to thank Dr Jing Yao Leong and Suzan Saidin for their assistance in sorting the immune subsets. Finally, the authors would also like to thank Insight Editing London for language editing of this manuscript prior to submission.

Contributors YHL and CJL performed most experiments, analysed the data and prepared the manuscript; LP analysed the NGS data; LL assisted in CyTOF data acquisition; CC performed RNA sequencing experiments; JY assisted in immune subsets sorting; MW performed univariate and multivariate analyses; TKHL and JY (pathologists) prepared and provided tissue samples; HCT (oncologist) advised on patient recruitment and clinical analysis; SYL, CYC, BKPG and AC (surgeons) assisted in patient recruitment and provided samples; $\mathrm{MH}$ (Zurich) and IOLN (Hong Kong) provided samples from overseas cohorts; PC provided clinical advice, recruited and provided samples for most patients; SA designed the study pipeline and assisted in writing the manuscript; $V C$ designed the studies, analysed the data and wrote the manuscript.

Funding This work was supported by the National Medical Research Council, Singapore (ref numbers: MOHIAFCAT2001, TCR15Jun006, CIRG16may048, CSAS16Nov006, NMRC/STaR/020/2013, CIRg13nov032, NMRC/MOHIAFCAT1-6003 and NMRC/MOHIAFCAT2/005/2015), the A*STAR Biomedical Research CouncilEconomy Development Board (BMRC-EDB IAF: IAF311020 and SPF2014/005) and Duke-NUS and SingHealth. MH was supported by an ERC COG grant (HepatoMetaboPath) and the SFB 179 and 209.

Competing interests None declared.

Patient consent Obtained.

Ethics approval Centre IRB.

Provenance and peer review Not commissioned; externally peer reviewed.

Open access This is an open access article distributed in accordance with the Creative Commons Attribution Non Commercial (CC BY-NC 4.0) license, which permits others to distribute, remix, adapt, build upon this work non-commercially, and license their derivative works on different terms, provided the original work is properly cited, appropriate credit is given, any changes made indicated, and the use is non-commercial. See:http://creativecommons.org/licenses/by-nc/4.0/.

(c) Article author(s) (or their employer(s) unless otherwise stated in the text of the article) 2019. All rights reserved. No commercial use is permitted unless otherwise expressly granted.

\section{REFERENCES}

1 El-Serag HB. Epidemiology of viral hepatitis and hepatocellular carcinoma. Gastroenterology 2012;142:1264-73.

2 El-Serag HB. Hepatocellular carcinoma. N Engl J Med 2011;365:1118-27.

3 Cai L, Zhang Z, Zhou L, et al. Functional impairment in circulating and intrahepatic NK cells and relative mechanism in hepatocellular carcinoma patients. Clin Immunol 2008;129:428-37

4 Wang F, Jing X, Li G, et al. Foxp3+ regulatory T cells are associated with the natural history of chronic hepatitis $B$ and poor prognosis of hepatocellular carcinoma. Liver Int 2012;32:644-55.

5 Fridman WH, Pagès F, Sautès-Fridman C, et al. The immune contexture in human tumours: impact on clinical outcome. Nat Rev Cancer 2012;12:298-306.

6 Vandeven N, Nghiem P. Pathogen-driven cancers and emerging immune therapeutic strategies. Cancer Immunol Res 2014;2:9-14.

7 Greten TF, Wang XW, Korangy F. Current concepts of immune based treatments for patients with HCC: from basic science to novel treatment approaches. Gut 2015:64:842-8

8 Hodi FS, O'Day SJ, McDermott DF, et al. Improved survival with ipilimumab in patients with metastatic melanoma. N Engl J Med 2010;363:711-23.
9 Rizvi NA, Hellmann MD, Snyder A, et al. Cancer immunology. Mutational landscape determines sensitivity to PD-1 blockade in non-small cell lung cancer. Science 2015;348:124-8.

10 Van Allen EM, Miao D, Schilling B, et al. Genomic correlates of response to CTLA-4 blockade in metastatic melanoma. Science 2015;350:207-11.

11 Melero I, Rouzaut A, Motz GT, et al. T-cell and NK-cell infiltration into solid tumors: a key limiting factor for efficacious cancer immunotherapy. Cancer Discov 2014:4:522-6.

12 Sia D, Jiao Y, Martinez-Quetglas I, et al. Identification of an Immune-specific Class of Hepatocellular Carcinoma, Based on Molecular Features. Gastroenterology 2017;153:812-26.

13 Thorsson V, Gibbs DL, Brown SD, et al. The Immune Landscape of Cancer. Immunity 2018;48:e14:812-30

14 El-Khoueiry AB, Sangro B, Yau T, et al. Nivolumab in patients with advanced hepatocellular carcinoma (CheckMate 040): an open-label, non-comparative, phase 1/2 dose escalation and expansion trial. Lancet 2017:389:2492-502.

15 Lavin Y, Kobayashi S, Leader A, et al. Innate Immune Landscape in Early Lung Adenocarcinoma by Paired Single-Cell Analyses. Cell 2017;169:e17:750-65.

16 Chew V, Lai L, Pan L, et al. Delineation of an immunosuppressive gradient in hepatocellular carcinoma using high-dimensional proteomic and transcriptomic analyses. Proc Nat/ Acad Sci U S A 2017;114:E5900-E5909.

17 Chew V, Lee YH, Pan L, et al. Immune activation underlies a sustained clinical response to Yttrium-90 radioembolisation in hepatocellular carcinoma. Gut 2019;68:335-46

18 Chew V, Tow C, Teo M, et al. Inflammatory tumour microenvironment is associated with superior survival in hepatocellular carcinoma patients. J Hepatol 2010:52:370-9.

19 Lai L, Ong R, Li J, et al. A CD45-based barcoding approach to multiplex masscytometry (CyTOF). Cytometry A 2015;87:369-74.

20 Shekhar K, Brodin P, Davis MM, et al. Automatic Classification of Cellular Expression by Nonlinear Stochastic Embedding (ACCENSE). Proc Natl Acad Sci U S A 2014;111:202-7.

21 Anders S, Pyl PT, Huber W. HTSeq-a Python framework to work with high-throughput sequencing data. Bioinformatics 2015;31:166-9.

22 Ward ST, Li KK, Curbishley SM. A method for conducting suppression assays using small numbers of tissue-isolated regulatory T cells. MethodsX 2014;1:168-74.

23 Garnelo M, Tan A, Her Z, et al. Interaction between tumour-infiltrating B cells and T cells controls the progression of hepatocellular carcinoma. Gut 2017;66:342-51.

24 Robinson MD, McCarthy DJ, Smyth GK. edgeR: a Bioconductor package for differential expression analysis of digital gene expression data. Bioinformatics 2010;26:139-40

25 Seetharam A, Perrillo R, Gish R. Immunosuppression in Patients with Chronic Hepatitis B. Curr Hepatol Rep 2014;13:235-44.

26 Murai M, Turovskaya O, Kim G, et al. Interleukin 10 acts on regulatory T cells to maintain expression of the transcription factor Foxp3 and suppressive function in mice with colitis. Nat Immunol 2009:10:1178-84

27 Huang CT, Workman CJ, Flies D, et al. Role of LAG-3 in regulatory T cells. Immunity 2004:21:503-13.

28 Camisaschi C, Casati C, Rini F, et al. LAG-3 expression defines a subset of CD4(+) CD25(high)Foxp3(+) regulatory T cells that are expanded at tumor sites. I Immunol 2010;184:6545-51.

29 Shang B, Liu Y, Jiang SJ, et al. Prognostic value of tumor-infiltrating FoxP3+ regulatory T cells in cancers: a systematic review and meta-analysis. Sci Rep 2015;5:15179.

30 Aalaei-Andabili SH, Alavian SM. Regulatory T cells are the most important determinant factor of hepatitis B infection prognosis: a systematic review and metaanalysis. Vaccine 2012;30:5595-602.

31 Pallett LJ, Davies J, Colbeck EJ, et al. IL-2 ${ }^{\text {high }}$ tissue-resident T cells in the human liver: Sentinels for hepatotropic infection. J Exp Med 2017;214:1567-80

32 Zheng C, Zheng L, Yoo JK, et al. Landscape of Infiltrating T Cells in Liver Cancer Revealed by Single-Cell Sequencing. Cell 2017;169:e16:1342-56.

33 Sun C, Sun H, Zhang C, et al. NK cell receptor imbalance and NK cell dysfunction in HBV infection and hepatocellular carcinoma. Cell Mol Immunol 2015:12:292-302.

34 Chew V, Chen J, Lee D, et al. Chemokine-driven lymphocyte infiltration: an early intratumoural event determining long-term survival in resectable hepatocellular carcinoma. Gut 2012;61:427-38.

35 Waggoner SN, Kumar V. Evolving role of 2B4/CD244 in T and NK cell responses during virus infection. Front Immunol 2012;3:377.

36 Sun C, Fu B, Gao Y, et al. TGF- $\beta 1$ down-regulation of NKG2D/DAP10 and 2B4/ SAP expression on human NK cells contributes to HBV persistence. PLOS Pathog 2012:8:e1002594.

37 Hoffmann SC, Cohnen A, Ludwig T, et al. 2B4 engagement mediates rapid LFA-1 and actin-dependent NK cell adhesion to tumor cells as measured by single cell force spectroscopy. J Immunol 2011;186:2757-64.

38 Ju Y, Hou N, Meng J, et al. T cell immunoglobulin- and mucin-domain-containing molecule-3 (Tim-3) mediates natural killer cell suppression in chronic hepatitis B. $J$ Hepatol 2010;52:322-9. 
39 Li H, Wu K, Tao K, et al. Tim-3/galectin-9 signaling pathway mediates T-cell dysfunction and predicts poor prognosis in patients with hepatitis B virus-associated hepatocellular carcinoma. Hepatology 2012;56:1342-51.

40 Chaudhary B, Elkord E. Regulatory T Cells in the Tumor Microenvironment and Cancer Progression: Role and Therapeutic Targeting. Vaccines 2016;4:28.

41 Fu J, Xu D, Liu Z, et al. Increased regulatory T cells correlate with CD8 T-cell impairment and poor survival in hepatocellular carcinoma patients. Gastroenterology 2007:132:2328-39.

42 Sideras K, Biermann K, Verheij J, et al. PD-L1, Galectin-9 and CD8 ${ }^{+}$tumorinfiltrating lymphocytes are associated with survival in hepatocellular carcinoma. Oncoimmunology 2017;6:e1273309.
43 Djenidi F, Adam J, Goubar A, et al. CD8+CD103+ tumor-infiltrating lymphocytes are tumor-specific tissue-resident memory $T$ cells and a prognostic factor for survival in lung cancer patients. J Immunol 2015;194:3475-86.

44 Webb JR, Milne K, Watson P, et al. Tumor-infiltrating lymphocytes expressing the tissue resident memory marker CD103 are associated with increased survival in high-grade serous ovarian cancer. Clin Cancer Res 2014:20:434-44.

45 Franceschini D, Paroli M, Francavilla V, et al. PD-L1 negatively regulates CD4+CD25+Foxp3+ Tregs by limiting STAT-5 phosphorylation in patients chronically infected with HCV. J Clin Invest 2009;119:551-64.

46 Gupta PK, Godec J, Wolski D, et al. CD39 Expression Identifies Terminally Exhausted CD8+ T Cells. PLoS Pathog 2015;11:e1005177.
EDITOR'S QUIZ: GI SNAPSHOT

\section{Anorexia, vomiting and weight loss in a 22-year-old woman}

See page 803 for question

\section{ANSWER}

This patient has immunotherapy-related gastritis which presented 1 year after the start of treatment. Checkpoint inhibitor immunotherapy has revolutionised the management of metastatic melanoma, and is approved for renal cell and non-small cell lung cancer. ${ }^{12}$ Ipilimumab is an anti-CTLA-4 antibody and nivolumab inhibits PD-1. The resultant immune activation is non-specific, leading to several well-described GI immune-related adverse events (irAE) including colitis, hepatitis and pancreatitis. ${ }^{3}$ Gastroenterologists should be aware that immunotherapy can also cause an isolated gastritis in the absence of duodenitis, and without concomitant diarrhoea or steatorrhoea. The treatment of irAE gastritis is corticosteroids, which runs counter to conventional treatment of gastritis (of which one of the causes is oral steroids). Her symptoms responded completely within 24 hours of starting oral prednisolone $40 \mathrm{mg}$. At her next clinic visit, she had gained weight and was eating normally without pain or vomiting. This is the first description in the literature of isolated immunotherapy-related gastritis. The key learning points are: to recognise that gastritis can occur on immunotherapy; that the presentation can be delayed; and that, unlike other forms of gastritis encountered by gastroenterologists, irAE gastritis is steroid responsive. As per the management of irAE colitis, we suggest that steroids are weaned over a 2-month period.

\section{Vincent Ting Fung Cheung, ${ }^{1}$ Eve Fryer, ${ }^{2}$ Miranda J Payne, ${ }^{3}$ Oliver Brain}

${ }^{1}$ Translational Gastroenterology Unit, John Radcliffe Hospital, Oxford, UK ${ }^{2}$ Department of Histopathology, John Radcliffe Hospital, Oxford, UK ${ }^{3}$ Department of Oncology, Churchill Hospital, Oxford, UK

Correspondence to Dr Vincent Ting Fung Cheung, Department of Gastroenterology, John Radcliffe Hospital, Headley Way, Oxford, OX3 9DU, UK; vincent.cheung@doctors.org.uk

Contributors VTFC and OB drafted the manuscript. VTFC and EF prepared the figures. All authors reviewed and edited the final article.

Competing interests None declared.

Patient consent Obtained.

Provenance and peer review Not commissioned; externally peer reviewed.

Data sharing statement This is a case report and therefore the data generated are not suitable for sharing beyond that contained within the report. Further information can be obtained from the corresponding author.

(c) Author(s) (or their employer(s)) 2019. No commercial re-use. See rights and permissions. Published by BMJ.

\section{A Check for updates}

To cite Cheung VTF, Fryer E, Payne MJ, et al. Gut 2019;68:927.

Received 19 June 2018

Revised 10 July 2018

Accepted 14 July 2018

Published Online First 30 July 2018

Gut 2019;68:927. doi:10.1136/gutjnl-2018-317023

\section{REFERENCES}

1 Hodi FS, O'Day SJ, McDermott DF, et al. Improved survival with ipilimumab in patients with metastatic melanoma. N Engl J Med 2010;363:711-23.

2 Antonia SJ, López-Martin JA, Bendell J, et al. Nivolumab alone and nivolumab plus ipilimumab in recurrent small-cell lung cancer (CheckMate 032): a multicentre, openlabel, phase 1/2 trial. Lancet Oncol 2016;17:883-95.

3 Spain L, Diem S, Larkin J. Management of toxicities of immune checkpoint inhibitors. Cancer Treat Rev 2016;44:51-60. 